\title{
Combined-modality hypofractionated radiotherapy for elderly patients with glioblastoma: setting a new standard
}

\author{
"The standard of care for glioblastoma management for patients below \\ 70 years of age, has been defined by the EORTC 26981/22981 NCIC-CE3 \\ trial $[7]$, fondly referred to as the 'Stupp regimen'.'
}

First draft submitted: 18 April 2017; Accepted for publication: 20 April 2017; Published online: 15 June 2017

Keywords: chemotherapy $\bullet$ geriatrics $\bullet$ glioblastoma $\bullet$ radiotherapy $\bullet$ trials

Glioblastoma (GBM) is the most common malignant primary brain tumour. It disproportionately affects the older segment of the population, as approximately half of the patients with GBM are above 65 years $[1,2]$. Moreover, the aging of the baby boom generation foreshadows a 'silver tsunami' of GBM [1].

Overlapping problems complicate management decisions for elderly patients, whether or not they have GBM. These may include multiple comorbidities and poor physiologic reserves, polypharmacy, limited mobility, cognitive decline, and social and financial vulnerability [3]. In addition, survival among elderly patients with GBM has been consistently shorter than among younger patients, with a population based median survival of approximately 6 months $(\mathrm{m})$ [4]. It is unclear if this is due to more aggressive tumor biology [5], the use of less intense treatment approaches [3] or increased susceptibility to treatment toxicities [6].

The standard of care for GBM management for patients below 70 years of age, has been defined by the EORTC 26981/22981 NCIC-CE3 trial [7], fondly referred to as the 'Stupp regimen'. This international, randomized controlled trial compared concurrent and adjuvant temozolomide (TMZ) with conventionally fractionated radiation therapy (RT; $60 \mathrm{~Gy} / 30$ fractions) to $\mathrm{RT}$ alone
(60 Gy/30 fractions). The combined modality therapy (CMT) group had improved overall survival (OS) compared with the RT alone group (hazard ratio [HR]: 0.63 95\%; CI: $0.52-0.75 ; \mathrm{p}<0.001)$. The 2 -year OS were 27 and $10 \%$, respectively. The presence of $\mathrm{O}(6)$-methylguanine-DNA methyltransferase (MGMT) methylation greatly accentuated the survival benefit with TMZ. The 5-year analysis [8] was published in 2009 and investigated if the benefit of TMZ was seen in older patients. Patients between 60 and 70 years of age comprised $30 \%$ of the participants. The median survival for this subgroup was lower with CMT (10.9 vs $11.8 \mathrm{~m})$. An unplanned subgroup analysis showed that survival differences for the 65-70-years old cohort failed to reach statistical significance (HR: 0.78; 95\% CI: $0.5-1.24 ; p=0.29)$ [2]. This suggested that older patients derived less benefit from CMT, although it had to be interpreted with caution due to the small patient numbers.

Many elderly patients with GBM seen in daily clinical practice do not qualify for the 'Stupp regimen' because of their age. Unfortunately, elderly patients have been excluded from many randomized clinical trials, and hence there is a lack of consensus on how this group should be managed - especially with regard to CMT.

Across various cancer types [9-11], hypofractionated radiotherapy regimens have

\section{Balamurugan A}

Vellayappan*,1, Lia M Halasz ${ }^{2}$ Jonathan PS Knisely ${ }^{3}$, Eric L Chang $^{4}$ \& Simon S Lo ${ }^{2}$ 1 Department of Radiation Oncology, National University Cancer Institute Singapore, National University Hospital, Singapore

${ }^{2}$ Department of Radiation Oncology, University of Washington School of Medicine, Seattle, WA, USA

${ }^{3}$ Department of Radiation Medicine, Northwell Health \& Hofstra Northwell School of Medicine, Lake Success, NY, USA

${ }^{4}$ Department of Radiation Oncology, University of Southern California, Keck School of Medicine, Los Angeles, CA, USA

*Author for correspondence:

Tel.: +65 67723084

bala_vellayappan@nuhs.edu.sg 
been increasingly adopted. Hypofractionated regimens reduce resource utilization for the healthcare system, potentially reduce costs and reduce the time spent by the patient commuting to and at the hospital. For instance, for a patient with an expected survival of $7 \mathrm{~m}$, a regimen that is 3 weeks shorter would free up $10 \%$ of their remaining lifetime spent with hospital visits. Roa et al. [12] demonstrated the noninferiority of $40 \mathrm{~Gy} / 15$ fractions compared with $60 \mathrm{~Gy} / 30$ fractions for patients with GBM aged 60 years and over with Karnofsky Performance status (KPS) $\geq 50$. Standard fractionation and hypofractionation provided OS of 5.1 and 5.6 months, respectively $(\mathrm{p}=0.57)$. Moreover, fewer patients in the hypofractionated arm stopped treatment prematurely (10 vs $25 \%$ ), suggesting better tolerability. However, widespread adoption was blunted as the reported survival was shorter than expected, and many felt the included patients were not representative of everyday practice. More recently, shorter regimens such as $25 \mathrm{~Gy} / 5$ fractions [13] and $34 \mathrm{~Gy} / 10$ fractions [14] have shown to be equally effective in elderly and/or frail patients. However, it has to be noted that the definition of elderly has varied among these trials from above 60 [14], 65 [15] and 70 years [16].

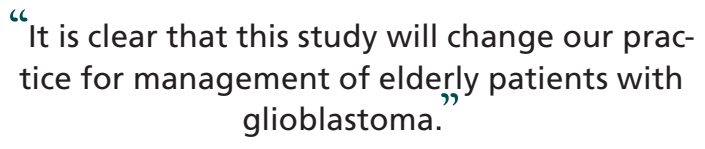
glioblastoma.

Compared with the 'Stupp regimen', hypofractionated RT regimens have been largely regarded as 'palliative' treatments. Hence, the aim of such treatments is to provide reasonable longevity as well as palliation without increasing treatment-related toxicity. To that end, many favored single-modality therapy as an approach with less toxicity. For example, the Nordic trial [14] compared three single modality treatments (60 Gy/30 fractions RT alone, TMZ alone $\left[200 \mathrm{mg} / \mathrm{m}^{2}\right.$ for 5 days every 28 days] and $34 \mathrm{~Gy} / 10$ fractions hypofractionated RT alone) in elderly patients with GBM. In this trial, patients with TMZ alone or hypofractionated RT alone had better outcomes than those treated with $60 \mathrm{~Gy}$.

In addition, regimens with fraction sizes larger than 2 Gy are seldom combined with concurrent chemotherapy for the same concerns of worse acute and late toxicities. However, late neurological toxicity is unlikely to clinically manifest in this group of patients with a short lifespan. Cao et al. [17] performed a retrospective study where patients $>60$ years $(\mathrm{n}=112)$ were treated with hypofractionated RT (40 Gy/15 fractions) with concurrent TMZ. Although a survival benefit was not demonstrable (median OS 6.9 vs 9.3 months for RT alone), only $9 \%$ of patients (from the combined modality arm) reported grade 3 or 4 hematological toxicity. Minniti et al. [18] conducted a prospective
Phase II trial involving 71 patients $>70$ years who were treated with hypofractionated RT (40 Gy/15 fractions) with concurrent TMZ and adjuvant TMZ (12 cycles). Patients from this cohort achieved a median survival of 12.4 months with a progression-free survival of 6 months. This study showed that this regimen is welltolerated with only $8 \%$ of patients having treatment interruption. Grade 3-4 toxicities occurred in $22 \%$ of patients, most of which were hematological.

Given the controversy over optimal treatment of elderly patients, Perry et al. are to be commended for designing and completing this international, randomized controlled trial (NCIC-CE6/EORTC 2606222061) [19]. From 2007 to 2013, 562 patients aged 65 years and above, with Eastern Cooperative Oncology Group (ECOG) performance status $\geq 2$ were randomized to hypofractionated RT $40 \mathrm{~Gy} / 15$ fractions with concurrent and adjuvant TMZ (12 cycles) versus RT alone ( $40 \mathrm{~Gy} / 15$ fractions). The arms were equally balanced in number and baseline characteristics. The median age was 73 years (range: $65-90$ years), with twothirds aged over 70 years. It has to be stressed that only patients deemed unsuitable for 'Stupp regimen' therapy were eligible for this study. Overall, $68.3 \%$ of patients underwent a partial or complete resection, and the remainder had biopsies only. The median OS was longer in the CMT arm (9.3 vs 7.6 months; HR: $0.67 ; 95 \%$ CI: $0.56-0.80 ; \mathrm{p}<0.001)$. The median PFS was also longer (5.3 vs 3.9 months; HR: 0.50 ; 95\% CI: $0.41-0.60$; $\mathrm{p}<$ $0.001)$.

MGMT status was available in $62.9 \%$ of participants, and promoter methylation was found in $46.6 \%$. Among these patients, median OS was much improved with CMT (13.5 vs 7.7 months; HR: 0.53 ; 95\% CI: $0.38-0.73 ; \mathrm{p}<0.001)$. An exploratory analysis suggested that combined modality in the MGMT methylated subgroup was associated with a persistent survival advantage at 24 months. In the unmethylated subgroup, median OS improvement was still clinically meaningful but just short of statistical significance (10.0 vs 7.9 months; HR: 0.75 ; 95\% CI: $0.56-1.01$; p $=0.055$ ). More importantly, although toxicity (mostly hematological and gastrointestinal) was worse in the CMT group, quality-of-life scores (measured through QLQ-C30 and QLQ-BN20) were similar in both arms. Treatment tolerability and adherence were high in both arms. More than $97 \%$ of patients completed radiotherapy and the median number of adjuvant TMZ cycles was five.

Patients were stratified at randomization according to their age groups: $65-70$ years, $71-75$ years, 75 years and above. It was surprising that the treatment effect was more pronounced in the older age groups, although this failed to reach statistical significance $(\mathrm{p}=0.06$ for 
interaction). This may be due to a selection bias, as only patients, including the 65-70 years subgroups, who were deemed unsuitable for 'Stupp regimen' were eligible for this study. As such, this group may have inherently worse outcomes from the outset.

$40 \%$ of the patients received some type of systemic therapy on progression, and the proportion was equal across the arms. More patients received salvage TMZ from the RT only arm. Patients from the CMT arm received agents other than TMZ, such as lomustine or bevacizumab. It is nearly impossible to determine whether these treatments were associated with longer survival, or whether the longer survival provided more opportunity for these patients to receive salvage systemic therapy.

It is clear that this study will change our practice for management of elderly patients with GBM. The treatment regimen used in this study is effective and tolerable and offers a meaningful benefit to older patients. However, there are still many unanswered questions:

- How does 40 Gy/15 fractions and TMZ compare to $60 \mathrm{~Gy} / 30$ fractions + TMZ?

- As in the 'Stupp regimen', we are still unsure if most of the benefit comes from the concurrent portion of TMZ or the adjuvant portion of TMZ - clarifying this may guide us in choosing the 'minimum required treatment' for patients unable to tolerate both concurrent and adjuvant phases;

\section{References}

1 Ostrom QT, Gittleman H, Fulop J et al. CBTRUS Statistical Report: primary brain and central nervous system tumors diagnosed in the United States in 2008-2012. Neuro Oncol. 17(Suppl. 4), iv1-iv62 (2015).

2 Laperriere N, Weller M, Stupp R et al. Optimal management of elderly patients with glioblastoma. Cancer Treat. Rev. 39(4), 350-357 (2013).

3 Paszat L, Laperriere N, Groome P, Schulze K, Mackillop W, Holowaty E. A population-based study of glioblastoma multiforme. Int. J. Radiat. Oncol. Biol. Phys. 51(1), 100-107 (2001).

4 Kita D, Ciernik IF, Vaccarella $S$ et al. Age as a predictive factor in glioblastomas: population-based study. Neuroepidemiology 33(1), 17-22 (2009).

5 Wiestler B, Claus R, Hartlieb SA et al. Malignant astrocytomas of elderly patients lack favorable molecular markers: an analysis of the NOA-08 study collective. Neuro Oncol. 15(8), 1017-1026 (2013).

6 Sijben AE, Mcintyre JB, Roldan GB et al. Toxicity from chemoradiotherapy in older patients with glioblastoma multiforme. J. Neurooncol. 89(1), 97-103 (2008).
- Can we do away with RT altogether for patients with MGMT methylation? [14,15]

- Can we combine TMZ with other hypofractionated regimens such as $34 \mathrm{~Gy} / 10$ or $25 \mathrm{~Gy} / 5$ fractions?

Undoubtedly, further trials will be needed to answer these questions and guide our practice.

Last, age is just a number and can be regarded as a 'soft' factor when deciding therapy. Age has been emphasized in clinical trials, and is included in many prognostic scoring systems, due to its objectivity and ubiquity. Tumor molecular profiling, physiological age and performance status, may be more important to determine which patients may benefit from more aggressive treatment.

\section{Financial \& competing interests disclosure}

The authors have no relevant affiliations or financial involvement with any organization or entity with a financial interest in or financial conflict with the subject matter or materials discussed in the manuscript. This includes employment, consultancies, honoraria, stock ownership or options, expert testimony, grants or patents received or pending, or royalties.

No writing assistance was utilized in the production of this manuscript.

\section{Open access}

This work is licensed under the Creative Commons Attribution 4.0 License. To view a copy of this license, visit http://creativecommons.org/licenses/by/4.0/

7 Stupp R, Mason WP, Van Den Bent MJ et al. Radiotherapy plus concomitant and adjuvant temozolomide for glioblastoma. N. Engl. J. Med. 352(10), 987-996 (2005).

8 Stupp R, Hegi ME, Mason WP et al. Effects of radiotherapy with concomitant and adjuvant temozolomide versus radiotherapy alone on survival in glioblastoma in a randomised Phase III study: 5-year analysis of the EORTCNCIC trial. Lancet Oncol. 10(5), 459-466 (2009).

9 Whelan TJ, Pignol JP, Levine MN et al. Long-term results of hypofractionated radiation therapy for breast cancer. N. Engl. J. Med. 362(6), 513-520 (2010).

10 Van Gijn W, Marijnen CA, Nagtegaal ID et al. Preoperative radiotherapy combined with total mesorectal excision for resectable rectal cancer: 12-year follow-up of the multicentre, randomised controlled TME trial. Lancet Oncol. 12(6), 575-582 (2011).

11 Din OS, Harden SV, Hudson E et al. Accelerated hypofractionated radiotherapy for non small cell lung cancer: results from 4 UK centres. Radiother. Oncol. 109(1), 8-12 (2013).

12 Roa W, Brasher PM, Bauman G et al. Abbreviated course of radiation therapy in older patients with glioblastoma multiforme: a prospective randomized clinical trial. J. Clin. Oncol. 22(9), 1583-1588 (2004). 
13 Roa W, Kepka L, Kumar N et al. International atomic energy agency randomized Phase III study of radiation therapy in elderly and/or frail patients with newly diagnosed glioblastoma multiforme. J. Clin. Oncol. 33(35), 4145-4150 (2015).

14 Malmstrom A, Gronberg BH, Marosi C et al. Temozolomide versus standard 6-week radiotherapy versus hypofractionated radiotherapy in patients older than 60 years with glioblastoma: the Nordic randomised, Phase III trial. Lancet Oncol. 13(9), 916-926 (2012).

15 Wick W, Platten M, Meisner C et al. Temozolomide chemotherapy alone versus radiotherapy alone for malignant astrocytoma in the elderly: the NOA-08 randomised, Phase III trial. Lancet Oncol. 13(7), 707-715 (2012).

16 Keime-Guibert F, Chinot O, Taillandier L et al. Radiotherapy for glioblastoma in the elderly. N. Engl. J. Med. 356(15), 1527-1535 (2007).
17 Cao JQ, Fisher BJ, Bauman GS, Megyesi JF, Watling CJ, Macdonald DR. Hypofractionated radiotherapy with or without concurrent temozolomide in elderly patients with glioblastoma multiforme: a review of ten-year single institutional experience. J. Neurooncol. 107(2), 395-405 (2012).

18 Minniti G, Lanzetta G, Scaringi C et al. Phase II study of short-course radiotherapy plus concomitant and adjuvant temozolomide in elderly patients with glioblastoma. Int. J. Radiat. Oncol. Biol. Phys. 83(1), 93-99 (2012).

19 Perry JR, Laperriere N, O'callaghan CJ et al. Shortcourse radiation plus temozolomide in elderly patients with glioblastoma. N. Engl. J. Med. 376(11), 1027-1037 (2017). 